\title{
34
}

\section{Game of pursuit with zero stop probability}

\author{
H. S. Kang \\ 470 Carnegie Dr. \\ Milpitas, CA 95035, U.S.A.
}

\begin{abstract}
The problem of Hierarchical structure in communicating with distantly located objects is formulated as $\mathrm{M}$ player $\mathrm{N}$ player nonzero sum game. $\mathrm{A}$ index for smallest winning coalition is introduced. Behavior strategy for coalition formed for evasion, and mixed strategy for coalition formed for pursuit are obtained. Repeatability and Precesion are defined for their application in communicating with distant bio objects. Necessary conditions of reachability for distantly located object with zero stop probability is formulated as Bimatrix game. Solution of Stochastic Bimatrix game in finite countable space is obtained. It is shown that mixed strategy used by pursuer over infinitely large partition is also an optimum strategy for stochastic game.
\end{abstract}

\section{Keywords}

Stochastic Games, Decision Making, Hierarchical Structure, Space Technology.

\section{INTRODUCTION}

With recent advances in space technology there is increased effort to communicate with distantly located intelligent life in space. For its application in communicating with distantly located objects the terms Repeatability and Precision are defined in this writeup. Dynamic and Nondynamic components of system (Figure 1) are dependent on unknown factors (as current state of the object). Problem of Hierarchial structure in communicating with distantly located bio objects sharing common workspace is formulated as rectangular nonzero sum $\mathrm{M} \times \mathrm{N}$ player game. $A$ index measure to induce players to participate in coalition is introduced. Index (Wmin) for smallest Winning coalition is also introduced. Essential games considered in this article may have infinitely many imputations. For reasons of its simplicity saddel point solution for class of games with restricted imputation core (Kang, 1972) is obtained. Necessary conditions for distantly located object may be reachable are obtained. Class of games (Friedman, 1971) with zero stop probability are discussed. Solution of 
stochastic game over a infinitely large partition is complex (Chris, 1971) and is not discussed in this article. Game of pursuit with zero stop probability is formulated as Bimatrix game over a finite countable space. It is shown that mixed strategy used by pursuer over infinitely large partition is also an optimum strategy for stochastic game. Results are illustrated with two numerical examples. When location of a intelligent object is restricted to a regional area Geosynchronous Satellite may be employed to track the bio object.

\section{MATHEMATICAL FORMULATION}

Given a dynamic system defined by subgroups of Borel sets $\left(t, U_{i}, Y, U_{0}, X\right)$. State transition map for the system is given by $k \Delta t \otimes k \Delta t \otimes U_{i} \otimes Y \otimes U_{0} \otimes X-X, T: Y$ $\rightarrow X$ is given by $\left[(k+1) \Delta t, k \Delta t, U_{i}, Y, U_{0}, X\right]=T(Y)$, and inverse State transition map for the system is given by $k \Delta t \otimes k \Delta t \otimes U o \otimes X \otimes U_{i} \otimes Y \rightarrow Y, T-1: X>Y$ is given by $\left[(k+1) \Delta t, k \Delta t, U_{0}, X, U_{i}, Y\right]=T^{-1}(x)$. Here elements of Borel sets $\left(t, U_{i}, Y\right.$, $U_{0}, X$ ) are further defined as follows: $t: k t$ is a ordered abelian group for time Set. $X$ : is a abelian group of vectors with $k \Delta t \rightarrow X$ a homomorphism of abelian group of vectors with $T: Y \rightarrow X$. Abelian group of $X$ is regarded as reflexive over field $R$ with $X$ ($R^{n} . Y$ : is a abelian group of vectors with $k \Delta t->$ of reflexive y $(-Y$, a $m$ tuplet over field $R^{m}$ with $y\left(-R^{m} . U_{i}, U_{0}\right.$ : are the abelian group of external stimulants.

\section{DEFINITIONS}

REPEATABILITY: Set $\mathrm{S}$ of reflexive field $\mathrm{X}$ is regarded as a Repeatable field if for all $x$ (- $S$ following holds ; $\alpha \neq 0, \alpha x \in S$.

For the purpose of application in communicating with distantly located intelligent life, Repeatability is further defined as Topological characterization of object field as it returns to given previously known initial state at the end every day.

PRECISION : Ability of system to track the distantly located living object as it moves to previously known state during assigned workhours.

Precision is of significance in its applications to the concept of Repeatability.

\section{MULTI PLAYERS SHARING WORK SPACE}

In the following it is assumed $\mathbf{N}$ players (Figure 1) in Hierarchical Structure share common work space and are integrated to form a evasion Coalition. It is also assumed $M$ players of 

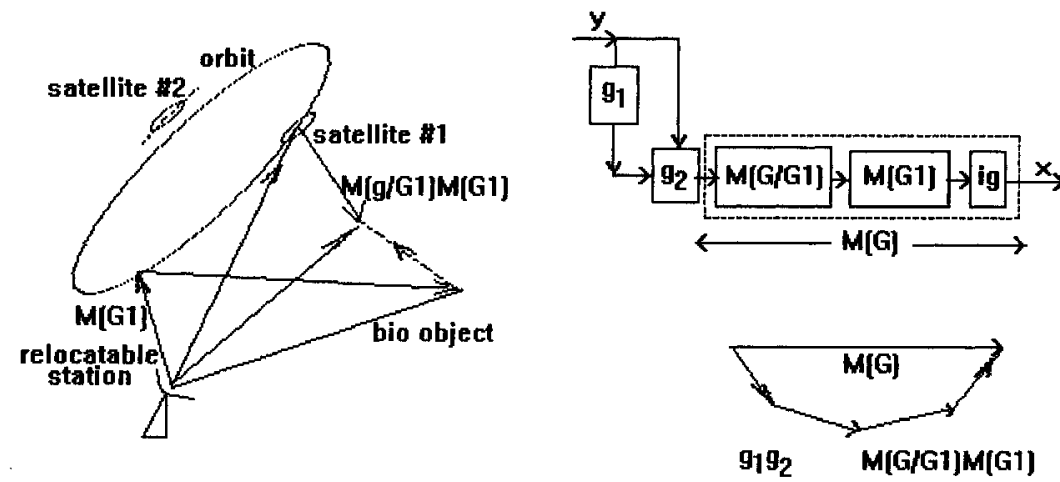

Figure 1. Compounded Transformation [satellite link]

pursuit coalition share the common work space (ref. Kang 1995). Further systems under consideration (ref. Kang 1995) may include relocatable control station and system interface board. Hierarchical structure payoff matrix may include Wmin, index for required smallest number of players combination to form a winning coalition. Modification of payoff matrix during game may serve the purpose of inducing evasion coalition players in pursuit coalition or for the purpose to exclude from evaders coalition. Hierarchical structure may consists of Upper Level the Coordinator, Intermediate level Organisors and Lower level Task Executors. Workspace for the game is topologically characterized as symetric transitive, further isomorphism of game is reflexive. Let probability distribution $P(y)$ for pursuit Coalition Game function $K_{j}(p)$ in product space $Y x X$ is given by $f_{y, x} K_{i}(y, x) d P(y) d x ; j=1,2$. In Hierarchical game structure multi level triplet of $\mathrm{N}$ players forming Coalition can be combined into larger game over a sum or product field.

\subsection{Imputation Core}

A game over Ring of field with product binary operation has payoff advantage over game with sum topology. It results in smaller value of Wmin the smallest number combination required to form a winning coalition. For the Hierarchical structure Essential game under consideration (Figure 2), class of core imputations are assumed as restricted.

THEOREM 4: Let $\eta, \mu, \xi$ be the solutions of game with product topology for Upper intermediate and Lower levels of Hierarchical Structure, and let $x_{j}$ be the solution of $K_{2}$ $\left(y_{i}, \eta_{j i}\right) \otimes K_{2}\left(y_{i}, \mu_{j i}\right) \otimes K_{2}\left(y_{j}, \xi_{j i}\right)$. Then Game function $K_{2}(y, x)$ for coalition and imputation for evasion are given by

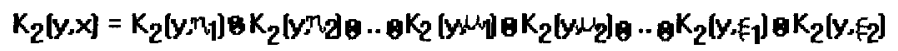




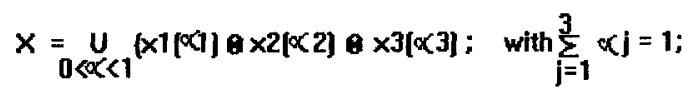

EXAMPLE: Consider $N \times N$ players game with evasion Coalition payoff $\left(E_{u}, E_{i}, E_{1}\right)$ of the Upper Intermediate and Lower levels (Figure 2) of Hirarchical Structure respectively. Let $P$ be the payoff for pursuit coalition. Using Theorem $4\left[E_{u}, E_{i}, E_{l}\right]_{\max }$ and $\left[E_{U}, E_{i}, E_{l}\right]_{\min }$ are the coalition imputation for evasion strategy. It may be verified that a payoff matrix modification may change imputations in core of Hierarchical structure to $\left[P, E_{i}\right]$ and $\left[P, E_{1}\right]$. Hyperplanes $H_{i}$ and $H_{j}$ are boundaries for permissible changes in Wmin index for evasion coalition imputation. In noncooperative game including games with possible defections, with multilevel Hierarchical structure, resulting index Wmin may not be within the permissible boundaries as specified by Hyperplanes. Further from Theorem 4 it can be verified (Figure 2) for a noncooperative games with possible defections imputation index may result in a lower payoff for evader coalition.

\subsection{N x N Players Game With Mixed Extension}

In Hierarchical structure information available to the Upper Intermediate and Lower levels is not directly available to other levels (Figure 2). The coordinator (dominant player) located at the Upper level acts as a generator for shared common workshape for other levels. Commands flow downwords and index for measure of performance flows upwords. Field of strategies available to Intermediate and Lower level players is dependent on strategy of dominant players.

The concept of Repeatability introduced in this paper considerably simplifies the analysis and design of systems for communicability. When Precision index of the game in the topology characterized by uncountable State space, is maximized over partitions, so formulated Game may have no solution. However, solution for the game exist on subgroup Ring of repeatable field. For Game so formulated Strategy for evader is characterized as Behavior strategy and Strategy for pursuer is mixed strategy. It can be shown mixed extension finite game may have a equilibrium saddle point solution.

\section{COMPOUNDED TRANSFORMATION IN REPEATABLE SYSTEMS:}

In the following a mathematical formulation of repeatable multistage process is given. Let group $G_{j}$ be decomposed to sequence of subgroups $G_{j i} i=1$, , n. Group can be represented by direct product of subgroup $G_{j}=X n_{i=1} G_{j j}$. Further it is assumed abelian subgroup $G_{j l}$ may be generated by generator $\left\langle g_{j l}>\right.$. For all $g_{j l}\left(-G_{j l}\right.$, the group $G_{j l}$; may be represented by zone $G_{j l}=\left(g_{j}, g_{j l}, ., g_{j l}\right)$. Let $m$ be the index and $r$ be the period for the Repeatability Measure $(m, r)$. Proposition 5.0 is stated in the following .

Proposition 5.0: Given a repeatable subgroup of group Ring $(R,+,$.$) over which$ evader employs the behavior strategy $\left(\eta_{j i}, \mu_{j i}, \xi_{j i}\right) ; i=1, \ldots, n$. There exists an optimal nonzero mixed strategy for the pursuer. 


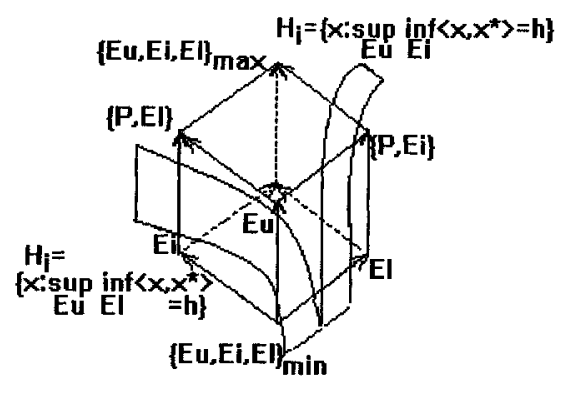

Figure 2. Bimatrix Game

[Hierarchical Structure]

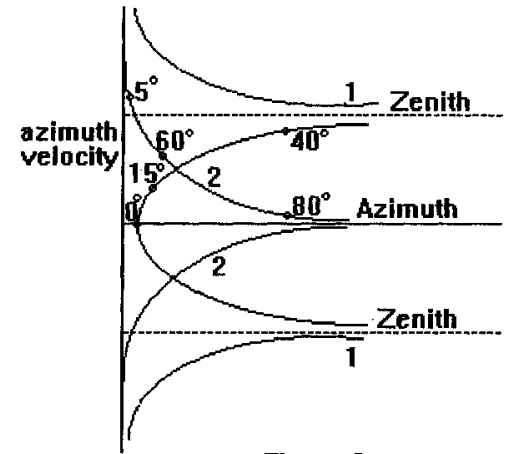

Figure 3.

1. zenith vs. azimuth vel.

2. azimuth vs. azimuth vel.

\subsection{Design of the Repeatable System}

In general system used for communicating with distantly located intelligent objects has space topology characterized by Repeatability measure $(m, r)$ and generator $\left\langle g_{j l}\right\rangle$, let $\psi$ be the annihilator polynomial for the system with $m=\operatorname{deg} \psi$. For repeatable system represented by countable field parameters system mapping matrix are given by

$$
F=\left|\begin{array}{rr}
0 & -\alpha m \\
1 & B
\end{array}\right| ; \quad B=[-\alpha m-1,-\alpha m-2, . ., \alpha 1]^{t}
$$

From proposition 5.0 the optimal solution for a repeatable system exists and it can be shown that optimal solution is obtained by minimizing precision index. Proposition 5.1 may be used to select the eigen vectors for the system. $\lambda_{0}$ is assumed to be known apriori.

Proposition 5.1: Let $v(t)$ and $v(s)$ be the values of the payoff matrix at time $t$ and $s$ . Let the strategy used by the pursuer is completely mixed. Let $\lambda_{t}$ and $\lambda_{s}$ be the eigen values of the matrix, then

$$
v_{t} / v_{s}=\lambda_{t} h_{s} ; \text { where } h_{s}=\lambda_{0}, v_{s}=v_{0} \text {, at } s=0 ;
$$

Precision index as given in equation (5) can be used to find self tuned controller for relocatable /mobil control station.

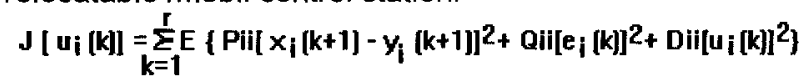

here $e_{i}(k)$ is the white gaussian noise, $P_{i i}, Q_{i i}$ and $D_{i i}$ are the weighting matrices. Necessary condition for distantly located object with Repeatability Measure $(m, r)$ be reachable is given by theorem 5.1 .

THEOREM 5.1. : Let the decomposable group $G_{j}$ consists of sequence of subgroups $\mathrm{G}_{\mathrm{ji}}, i=1$, , n. Further define a group Ring $(R(\mathrm{Gj}),+,$.$) . Let \mathrm{S}_{\mathrm{ji}}$ be the subset of subgroups $\mathrm{G}_{\mathrm{ji}}, i=1, ., n$. It is assumed following axioms are true: (i) $\phi \notin S_{j i},(i i) \alpha_{1 i}\left(-S_{j i}, \alpha_{2 i}\left(-S_{j i}\right.\right.$ 
implies $\alpha_{1 i} \cap \alpha_{2 i}\left(-S_{j i} i=1,, n,(i i i) \alpha_{1 i} \supset \alpha_{2 i}, \alpha_{2 i}\left(-S_{j i}\right.\right.$ implies $\alpha_{1 i}\left(-S_{j i}\right.$, (iv) For all $\times\left(-S_{j i}\right.$ implies $x+\delta x(-S j i,(v)$ Topological space $(x, k \Delta t)$ be Hausdroff Space. Then using sequence $x_{j i}\left(-S_{j i}, i=1, \ldots, n\right.$, the object in abelian $G j i$ is reachable, i.e. for all $x_{l}\left(-S_{j i}, \| x-\right.$ $S_{j i l}\|=<\| x-S_{j l} \|$.

EXAMPLE: To illustrate results stated above consider a Satellite Tracker/Telescope used for studying cesestial bodies (Figure 1a) with system dynamics given by equation (6). It is desired to maximize the reachable zone. System may be configured using compounded transformation (Figure 1b).

$(d Z / d H)=C 1 \operatorname{Sin}(A) ;(d A / d H)=C 2-C 3 \operatorname{Cot}(Z) \operatorname{Cos}(A)$

Here $Z$ and $A$ are the Zenith and Azimuth angles. $H$ is the hour angle mark of planet. It is assumed $Z(O)=Z O, A(0)=A O$. For the purpose of analysis precision index in $C^{2}[0, T]$ space (ref. Gaughan 1968) may further be simplified for maximizing tracking velocity. Frechet differential of equation (6) for maximum azimuth velocity is given by $(\mathrm{d} A / \mathrm{dH})=2{ }^{*} \mathrm{C} 1$ (Cos A/Sin2Z), From the results as plotted in Figure 3 it is seen azimuth velocity is very large for low values of Zenith. Azimuth velocity of Tracker/ Telescope tends to be very low as azimuth becomes orthogonal to its axis horizon i.e. when location of a intelligent object is restricted to a regional area Geosynchronous Satellite may be employed to track the bio object. To establish reachability (Theorem $5.1)$ product space topology may be employed for the system under consideration. From equations (6) we obtain

$S[d Z / d H]=0 ; \delta(d A / d H]=C 3[\cot [Z+\delta Z] \cos [A+\delta A]-\operatorname{Cot} Z \operatorname{Cos} A \quad(7)$

For given constrains it can be verified $\delta(\mathrm{dZ} / \mathrm{dH})=\delta(\mathrm{dAdH})=0$. Using convergence in product space as stated in Theorem 5.1, distantly located object in general may be continuously tracked.

\section{COMPOUNDED TRANSFORMATION}

Decomposition Lemma 6.0 for compounded transformation is stated below: Lemma 6.0 may be used to design and configure a relocatable Control Station/Satellite Link.

Lemma 6.0: Given a group $G_{j}$, with a sequence of subgroups $G_{j i}, i=1, ., n, M\left(G_{j i}\right)$ can be decomposed as cascade collection of components given by equation ( 8 ).

$M\left[G_{j i}\right]=M\left[G_{i} / G_{i-1} . . G_{1}, G_{i-1} / G_{i-2} . . G, G_{i-2} / G_{i-3} . . G, \ldots, G_{2} / G_{1}\right]$

[8]

When $G_{i j} ; i=1$, , n are semigroups, equation (8) could further be simplified. Lemma 6.0 may be used for Figure 1 to obtain decomposition of Satellite Link frame system. Decomposed system can be used to design and configure a relocatable Control Station / Satellite Link frame (Figure 1). 


\section{STOCHASTIC GAME WITH ZERO STOP PROBABILITY :}

Recently Kang (1995) has analyzed process for extending reachable zone and use of relocatable control station for the purpose. In this section a pursuit game with zero stop probability is formulated as a Bimatrix game.

\subsection{Statement of Stochastic Bimatrix Game Problem}

It is assumed generator for sequence of subgroup for Group $G$ consists of Borel sets $<T \times U_{i} \times Y \times U_{0} \times X>$. We assume performance index for the system is given by $W\left(p, u_{i}, u_{0}\right)$ where $u_{j}, u_{0}$ are given by axioms stated above. Given the current $(i$ th) state, let $p(j / i)$ be the conditional probability of transition to $j$ th state at following stage. Assume $C_{j}$ be the return for the $j$ th state, $\beta$ be the discount factor, $S_{p}$ be the probability of stop, and $(m, r)$ is the Repeatability measure. Limiting average payoff at $j$ th state is given by equations (10) and conditional probability of exit from repeatable zone is given by equation (11)

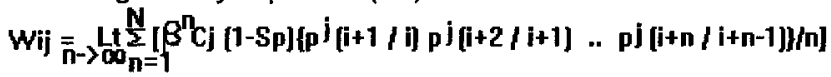

$$
W i j=\sum_{n-\infty}^{L+\infty} \sum_{n=1}^{N}\left[\beta^{n} \operatorname{Cj}[1-S p]\left\{p(j i]^{n / m}\right] / n\right]
$$

$$
p\left[j i j=p^{j}[i+1 / i] p^{j}(i+2 i+1) \ldots p^{j}(i+m j i+m-1)\right.
$$

\subsection{Conditional Probability Distribution Matrices}

Conditional probability matrices for the State of distant bio objects and for relocatable control station at $i$ th stage are given in tables (7.1-7.2). It is assumed measurements for evader are available to pursuer. It is also assumed evader has no access to the measurements for the pursuer state. It is assumed due to restrictions placed by the system dynamics, relocatable control station may only move to neighboring grid node during a state transition. The probability $\mathrm{p}(\mathrm{y}(\mathrm{i}+1) / \mathrm{x}())$ of pursuer

\begin{tabular}{|c|c|c|c|c|c|c|c|c|c|}
\hline & Table 7. & & & & & & able 7.2 & & \\
\hline$y(i-1)$ & $y(i)$ & $y(i+1)$ & & $x(i-1)$ & $x(i)$ & $x(i+1)$ & $x(i-1)$ & $x(i)$ & $x(i+1)$ \\
\hline$x(i-1) \mid p(i-1 / i-1)$ & $\mid p(i-1 / i)$ & 0 & 1 & $|U(i-1)|$ & $U(\mathrm{i}-1$ & $\mathrm{U}(\mathrm{i}-1)$ & $\mid p(i-1 / i-1)$ & $p(i / i-1)$ & $\mid p(i-1 / i-1)$ \\
\hline$x(i) \cdot \mid p(i-1 / i)$ & | $p(i / i)$ & | $p(i+1 / i)$ & I & $\mathrm{U}(\mathrm{i})$ & $U(i)$ & $U(i)$ & $p(i-1 / i)$ & | $p(i / i)$ & $\mid p(i+1 / i)$ \\
\hline $\begin{array}{l}x(i+1) \mid 0 \\
p(i+1 / i+1) \mid\end{array}$ & $\mathrm{p}(\mathrm{i}+1 / \mathrm{i})$ & $p(i+1 / i+1$ & & $|U(i+1)|$ & $\mathrm{U}(\mathrm{i}+1$ & $U(i+1)$ & $\mid p(i-1 / i+1)$ & l p(i/i+ & \\
\hline
\end{tabular}
state is given by Table 7.1 .

The probability distribution of evader strategy and the probability of evader state as predicted by prsuer at $i$ th state is given by table 7.2 and the probability $p(x(i+1))$ of evader state as estimated by pursuer is given by equation (14) 
$p\{x(i+1)\}=p\{x(i+1) / x i\} p\{x i / x(i-1) x(i-2) \quad . . x(0)\}$

Equations (10-14) can be used to compute mixed strategy for the pursuer and Behavior strategy for the evader. Payoff for game can be computed using equation (10).

\section{CONCLUSIONS}

This article addresses the problem of communicating with intelligent life in distant space. Problem of Hierarchical structure in communicating with distant bio objects is formulated in product space. Necessary conditions that distant bio object may be reachable are obtained. It is shown that given system may be decomposed as cascade collection of decomposed sequence of subgroups. Decomposed sequence so obtained is further used in compounded transformation for solution of process of communicating with distant bio object and implementing a relocatable control station / Satellite Link for system. The problem is formulated as Bimatrix game with zero stop probability in finitely countable space. It is shown saddle point equilibrium obtained over large partition is also the solution for the equivalent stochastic game. Results are illustrated with two numerical examples.

\section{REFERENCES}

Kang, H.S. (1972) Optimal Stochastic Control Via Differential Games. Hawaii Systems Science Confererance, Hawaii, U.S.A.

Tsokos, C.P., and Padsett, W.J. (1971) Random Integral Equation with Applications to Stochastic Systems. Springer Verlag, Berlin.

Gaughan, E. (1968) Introduction to Analysis. Bokks/Cole Publication Cp, Div. of Wadisworth Publishing Co, Belmont.

Friedman, A. (1971) Differential Games. Wieley- Interscience, John Wieley \& Sons, London. Kang, H.S.. (1970) On the Lyopunov Design of Systems with Zeros in Right Half Plane. I.E.E.E. Transactions on Automatic Control, Vol 2, 1970, U.S.A.

Kang, H.S. (1995) Canonical Transformation in Modelling Biosystems. IFAC - 17th IFIP TC7. Conference on Systems Modelling and Otimization, July 1995, Prague, Czech Rep. 